\title{
Erratum zu: Symptomatische Behandlung nach Schlaganfall
}

\section{Erratum zu:}

Dtsch Z Akupunkt 2020

https://doi.org/10.1007/s42212-020-00260-w

Im Originalbeitrag sind leider die Tabellen in der Einleitung sowie das „Fazit“ entfallen. Wir bitten um Beachtung der hier aufgeführten Ergänzung und des aktualisierten Interessenkonflikts.

\section{Einleitung}

Schlaganfälle sind eine der weltweit häufigsten Ursachen für körperliche, aber auch seelische Einschränkungen. Neben einer leitliniengemäßen Behandlung der Ursachen des Schlaganfalls (ischämisch oder hämorrhagisch) liegt der Fokus der Behandlung in der Stroke Unit und anschließenden Rehabilitation auf der Wiederherstellung der verlorenen Funktionen. Physio- und Ergotherapie sowie Logopädie nehmen eine zentrale Rolle ein [12]. Das Outcome der Patienten ist stark unterschiedlich, die Gründe hierfür sind noch unverstanden. Häufig bleiben dauerhafte Einschränkungen zurück. Insbesondere in China werden Akupunktur und verwandte Verfahren ergänzend in den Therapieplan aufgenommen und zunehmend auch systematisch in klinischen Studien erforscht.

Im Folgenden werden die Ergebnisse der aktuellsten Metaanalysen zum Einsatz der Akupunktur in der Schlaganfallrehabilitation im Sinne eines Rapid Review detailliert vorgestellt. Im Zeitraum von Juli bis November 2019 wurden in einer PubMedLiteraturrecherche 11 relevante Metaanalysen bzw. Übersichtsarbeiten über Metaanalysen identifiziert (Zusammenfassung siehe Tab. 1 und 2). Die folgende Übersicht für die Kitteltasche greift die folgenden 5 relevanten Rehabilitationsindikationen auf, wobei jeweils nur die aktuellsten Publikationen (in Summe 6) berücksichtigt wurden:

1. Schulter-Arm-Syndrom,

2. Depression,

3. Aphasie,

4. Dysphagie,

5. Aktivitäten des täglichen Lebens (ADL).

Die Online-Version des Originalartikels ist unter https://doi.org/10.1007/ s42212-020-00260-w zu finden.

Deutsche Zeitschrift für Akupunktur 2020 • 63 (3): 139-141

https://doi.org/10.1007/s42212-020-00274-4

Online publiziert: 29. Mai 2020

(c) Springer Medizin Verlag GmbH, ein Teil von Springer Nature 2020
Fazit

Die Evidenz für den Einsatz der Akupunktur ist trotz vielversprechender Ansätze noch nicht überzeugend. Wesentlich verantwortlich hierfür ist die Herkunft nahezu aller zugrundeliegenden klinischen Studien aus China sowie deren zum Teil niedrige methodologische Qualität.

Trotzdem kann die Akupunktur als ein sicheres Verfahren im Einsatz zusätzlich zur Standardtherapie gerechtfertigt werden.

Literatur

1. Liu S, Zhang CS, Cai Y et al (2019) Acupuncture for post-stroke shoulder-hand syndrome: a systematic review and meta-analysis. Front Neurol. https:// doi.org/10.3389/fneur.2019.00433

2. Zhang X-Y, Li Y X X, LiU D-L et al (2019) The effectiveness of acupuncture therapy in patients with post-stroke depression: an updated meta-analysis of randomized controlled trials. Medicine 98:e15894. https://doi.org/10.1097/ MD.0000000000015894

3. Li X, Wang J, Xu A et al (2018) Clinical effects and safety of electroacupuncture for the treatment of post-stroke depression: a systematic review and meta-analysis of randomised controlled trials. Acupunct Med 36:284-293. https://doi.org/10.1136/ acupmed-2016-011300

4. Zhang B, Han Y, Huang X et al (2019) Acupuncture is effective in improving functional communication in post-stroke aphasia. Wien Klin Wochenschr 131:221-232. https://doi.org/10.1007/s00508-019-1478-5

5. Li L, Deng K, Qu Y (2018) Acupuncture treatment for post-stroke dysphagia: an update meta-analysis of randomized controlled trials. Chin J Integr Med 24:686-695. https://doi.org/10.1007/s11655-018-3005-3

6. Garcia-Rudolph A, Sánchez-Pinsach D, Salleras EO, Tormos JM (2019) Subacute stroke physical rehabilitation evidence in activities of daily living outcomes. Medicine. https://doi.org/10.1097/MD.0000000000014501

7. Hung CY, Wu X, Chung VC et al (2019) Overview of systematic reviews with metaanalyses on acupuncture in post-stroke cognitive impairment and depression management. Integr Med Res 8:145-159. https://doi.org/10.1016/j.imr.2019.05.001

8. Chau JPC, LO SHS, Yu X et al (2018) Effects of acupuncture on the recovery outcomes of stroke survivors with shoulder pain: a systematic review. Front Neurol 9:30. https://doi.org/10.3389/fneur.2018.00030

9. Liu A-J, Li J-H, Li H-Q et al (2015) Electroacupuncture for acute Ischemic stroke: a meta-analysis of randomized controlled trials. Am J Chin Med 43:1541-1566. https://doi.org/10.1142/S0192415X15500883

10. Xin Z, Xue-Ting L, De-Ying K (2015) GRADE in systematic reviews of acupuncture for stroke rehabilitation: recommendations based on high-quality evidence. Sci Rep 5:16582. https://doi.org/10.1038/srep 16582

11. Kong JC, Lee MS, Shin B-C et al (2010) Acupuncture for functional recovery after stroke: a systematic review of sham-controlled randomized clinical trials. CMAJ 182:1723-1729. https://doi.org/10.1503/cmaj.091113

12. Teasell Robert W, Lalit K (2005) What's new in stroke rehabilitation. Stroke 36:215-217. https://doi.org/10.1161/01.STR.0000153061.02375.04 

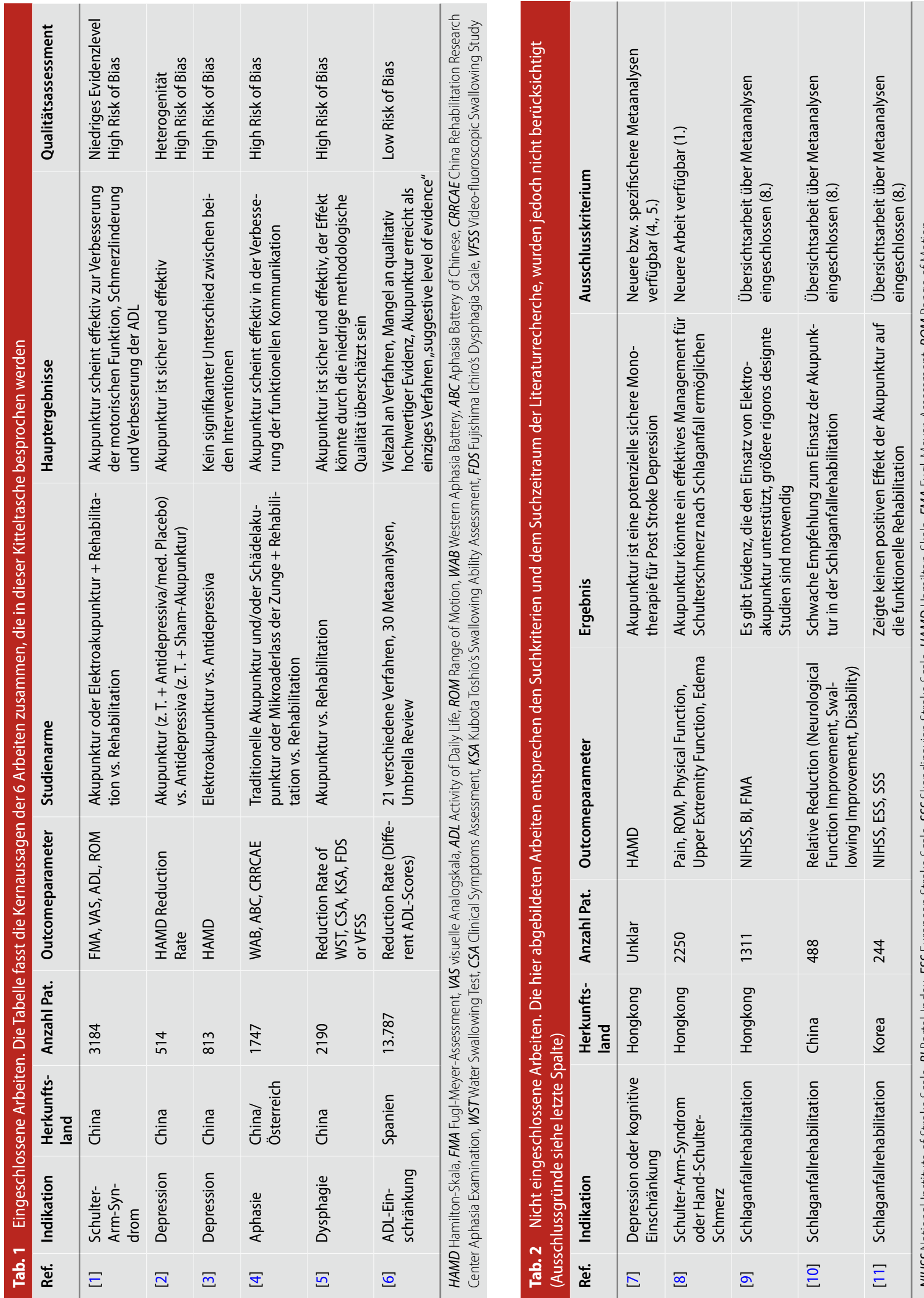

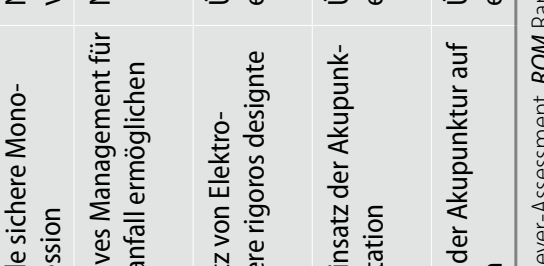

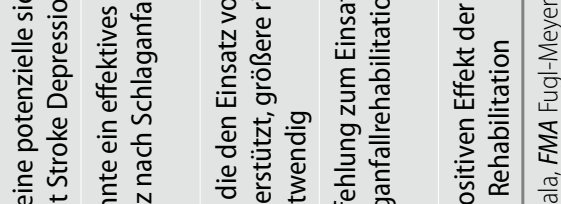

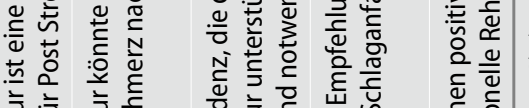

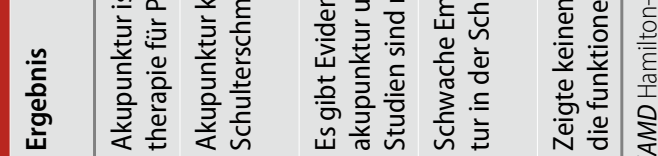

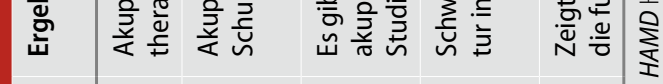
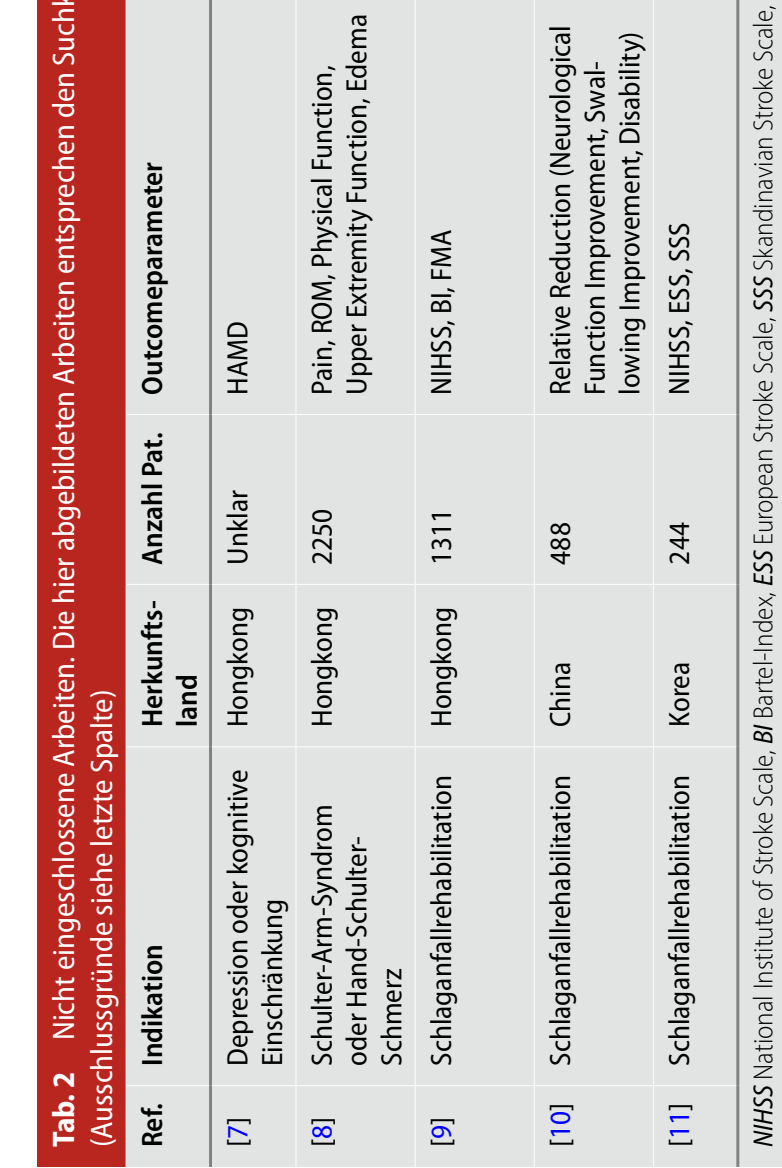


\section{Fachnachrichten}

\section{Korrespondenzadresse}

\section{Malte Fischer}

Innere Medizin mit Schwerpunkt Geriatrie,

Klinik Hennigsdorf

Marwitzer Str. 91, 16761 Hennigsdorf, Deutschland

malte94@gmx.de

Interessenkonflikt. M. Fischer gibt an, dass kein Interessenkonflikt besteht. Die Grundlagen zu diesem Artikel sowie zu dieser Serie wurden im Rahmen eines ausgeschriebenen und honorierten Auftrags des Wissenschaftszentrums der DÄGfA erarbeitet.

\section{Akupunktur bei rezidivierenden Harnwegsinfektionen von Frauen}

Chinesische und australische Wissenschaftler führten ein Review mit Metaanalyse zu Akupunktur und verwandter Techniken bei unkomplizierten rezidivierenden Harnwegsinfektionen von Frauen durch. Sie fanden zu dieser Fragestellung in verschiedenen internationalen und chinesischen Datenbanken 5 randomisierte kontrollierte Studien mit 341 Teilnehmerinnen.

Die methodische Studienqualität und Evidenzstärke wurde als gering bis mäßiggradig beurteilt. Die Chancen auf eine Heilung stellten sich mit einer komplexen Behandlung einschließlich Akupunktur größer dar als mit Antibiotika (3 Studien mit 170 Teilnehmerinnen, $R R=1,82,95 \% \mathrm{Cl}$ 1,31 bis 2,81). Das Rezidivrisiko war mit Akupunktur geringer als ohne Behandlung (2 Studien mit 135 Teilnehmerinnen, RR 0,39, 95\% Cl 0,26 bis 0,58 ) oder unter Sham-Akupunktur (1 Studie, 53 Teilnehmerinnen, RR $0,45,95 \% \mathrm{Cl} 0.22$ bis 0,92 ). Schlussfolgerung: Akupunktur scheint zur Behandlung und Prävention von rezidivierenden Harnwegsinfekten bei Frauen mit begrenzter Evidenz von Nutzen zu sein. Angesichts wachsender Antibiotikaresistenzen besteht ein Bedarf an qualitativ hochwertigen Studien zu nicht-medikamentösen Behandlungsverfahren wie Akupunktur.

Literatur:

Qin X, Coyle ME, Yang L, et al (2020) Acupuncture for recurrent urinary tract infection in women: a systematic review and meta-analysis. BJOG. doi: 10.1111/1471-0528.16315. Online ahead of print

Axel Wiebrecht 